8-20-2018

\title{
Combined Effect of Oxidative Treatment and Residual Alcohol on the Mechanics of a Multiwalled Carbon Nanotube Laden Interface
}

William Ivancic

Cleveland State University

Christopher L. Wirth

Cleveland State University

Follow this and additional works at: https://engagedscholarship.csuohio.edu/encbe_facpub

Part of the Chemical Engineering Commons

How does access to this work benefit you? Let us know!

\section{Repository Citation}

Ivancic, William and Wirth, Christopher L., "Combined Effect of Oxidative Treatment and Residual Alcohol on the Mechanics of a Multiwalled Carbon Nanotube Laden Interface" (2018). Chemical \& Biomedical Engineering Faculty Publications. 133.

https://engagedscholarship.csuohio.edu/encbe_facpub/133

This Article is brought to you for free and open access by the Chemical \& Biomedical Engineering Department at EngagedScholarship@CSU. It has been accepted for inclusion in Chemical \& Biomedical Engineering Faculty Publications by an authorized administrator of EngagedScholarship@CSU. For more information, please contact library.es@csuohio.edu. 


\title{
Combined effect of oxidative treatment and residual alcohol on the mechanics of a multiwalled carbon nanotube laden interface
}

\author{
William Ivancic, Christopher L. Wirth* \\ Chemical and Biomedical Engineering Department, Washkewicz College of Engineering, Cleveland State University, 2121 Euclid Ave., Cleveland, oH, 44115, United States
}
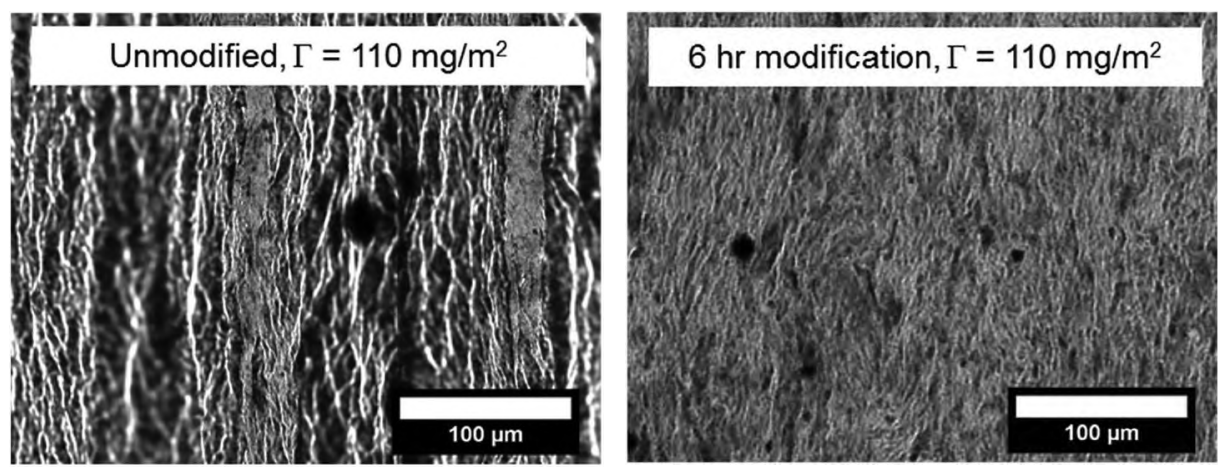

\section{Keywords:}

Carbon nanotubes

Particles at interfaces

Surface pressure

Particle wetting

Film stiffness
Single and multiwalled carbon nanotubes (SWCNT \& MWCNT) have been investigated over the past three decades because of their excellent properties, including their mechanical strength and large electrical and thermal conductivities. Incorporating CNTs into consumer and industrial products is challenging because of strong attractive interactions, heterogeneity, and lack of separation techniques for these nanomaterials. Further challenges include those associated with CNT interaction and adsorption to interfaces. In the present work, the properties of an air/water interface laden with industrial grade MWCNTs with systematically varied oxidative treatment in nitric acid were measured. The duration of treatment was varied and the surface pressure of the nanotube laden interfaces was measured. Results suggested that film stiffness increased with treatment duration in the presence of residual isopropyl alcohol. However, these measurements revealed that stiffness increased with compression number, suggesting that treatment duration had an indirect effect and compressive history was primarily responsible for changes in interfacial mechanics. Additionally, optical micrographs showed evidence of buckling in the films at low modification times. Relaxation of a compressed interface showed that MWCNTs with longer oxidative treatments induced longer relaxation times. These data suggest MWCNT films densify particleparticle contacts at higher modification times, with relaxation occurring through diffusive microstructural changes. One potential physical mechanism for such behavior is that MWCNT aggregate due to capillary interactions brought on by stronger adsorption to the interface in the presence of residual alcohol and longer oxidative treatment. 


\section{Introduction}

Carbon nanoparticles of varying structure and morphology are regularly found in applications, including consumer products, electrode materials, transistors, and medicine [1-4]. Carbon nanotubes, in particular, have been adopted as additives because of the particles' strength [5], high conductivity [6], and potential as a florescent tag $[7,8]$. Appropriate processing strategies need to be developed for effective integration of these materials into products [1], including fluid formulation, deposition, and drying. Carbon nanotubes will often, either intentionally or unintentionally, interact with solid/fluid or fluid/ fluid interfaces during these processing steps. For instance, LangmuirBlodgett coating, where nanoparticles are initially located at a fluid/ fluid interface and then deposited onto a moving substrate impinging on that interface, is regularly utilized to deposit nanoparticles onto substrates [9-13]. The interfacial microstructure and dynamic ordering of nanoparticles in response to capillary attraction after deposition are critical considerations for fabricating a desirable thin film with Langmuir-Blodgett. Utilization of these processing steps necessitate understanding interfacial properties of solid carbon nanomaterials near to or adsorbed to a fluid/solid or fluid/fluid interface.

Solid nano- to microscale particles will impart complex mechanical and rheological properties to an interface depending on the surface chemistry, size, and shape of the particle [14-17]. Solid particles may have a pinning energy exceeding $>10^{6} \mathrm{kT}$ when at a fluid/fluid interface. In addition, particle-particle interactions depending on either the separation distance or relative orientation will induce microstructure that imparts potentially useful interfacial rheological behavior to an interface. The strong pinning energy and microstructurally mediated rheological properties typically makes solid stabilized foams and emulsions extremely stable [18-21]. These general properties of solid stabilized interfaces, along with the favorable functionality of CNTs makes CNTs an attractive option for not only stabilizing multiphase materials, but also fabricating functional emulsions and foams.

Previous work on the adsorption and mechanics of carbon nanomaterials to a fluid/fluid interface has shown a wide parameter space [22-27]. Particle dimension, shape, size, surface chemistry, sub-phase $\mathrm{pH}$, and morphology could all play a role in microstructurally mediated interfacial mechanics. For instance, asphaltenes regularly found in the oil and gas industry impart complex mechanical and interfacial phenomena that range from interfacial tension to microstructurally driven phenomena [22-24]. Furthermore, the mechanics of non-functionalized and functionalized CNTs adsorbed to an air/water interface was measured, along with contact angle and microstructure. The authors found that a strong oxidizing environment caused the contact angle for the CNTs to transition from non-wetting to wetting. This oxidative treatment impacted the mechanics of the interface, with the functionalized CNTs imparting a higher elasticity to the water/air interface. Interfacial tension should depend only on the surface concentration of material and the exchange of molecules or particles to and from the interface. However, other microstructural stresses will often arise when large molecules or solid particles form appreciable microstructure that manifests as a solid-like skin. Identifying and delineating between these contributions to stress is crucial to determining the physical picture behind interfacial rheological measurements.

Work presented herein seeks to elucidate these phenomena for industrial grade multiwalled carbon nanotubes (MWCNT) at an air/water interface. Industrial grade MWCNTs are an economical candidate for integrating into consumer products or industrial processes. Specifically, the effect of oxidative treatment and residual alcohol (isopropyl alcohol (IPA)) on the mechanics of the MWCNT laden interface was measured. Following oxidative treatment and addition of material to the interface, optical microscopy and surface pressure measurements were conducted during dilatational deformation of an interface laden with MWCNTs. IPA and oxidative treatment cooperatively induced variations in mechanics depending on the duration of MWCNT oxidative treatment.
MWCNTs in the presence of IPA and treated for a longer duration in an oxidative environment achieved a film with higher stiffness, when compared with MWCNTs that had been treated for a shorter period of time. These results are consistent with a mechanism in which IPA enhances wetting of MWCNTs at an interface depending on oxidative treatment, thereby increasing capillary attraction. Increasing the net attractive particle-particle interactions makes the resulting film stiffer and increases the time necessary for relaxation following deformation. Results summarized herein provide guidance as to how to formulate multiphase materials utilizing industrial grade MWCNTs.

\section{Experimental}

\subsection{Oxidative treatment of multi-walled carbon nanotubes}

Industrial grade MWCNTs were obtained and arrived dry from Nanolabs (Waltham, MA). As has been done previously [28], the MWCNTs were exposed to a strong oxidizing environment of nitric acid at elevated temperature. $1.0 \mathrm{~g}$ of dry MWCNTs were added to $200 \mathrm{~mL}$ of nitric acid (ACS reagent, $70 \%$, Sigma-Aldrich) in a $250 \mathrm{~mL}$ round bottom flask with condenser and stir bar. The round bottom flask was situated in a hot water bath with a set temperature of $60^{\circ} \mathrm{C}$. The round bottom flask provided a second access point that allowed for removing samples during the oxidative treatment. Aliquots of $20 \mathrm{~mL}$ of the MWCNT suspension were removed at 40,60, 120, 240, and $360 \mathrm{~min}$. These samples were immediately delivered into filter paper for vacuum filtration. The material was washed with a copious volume of water, during which the $\mathrm{pH}$ was measured. Ultra-pure water was used to remove excess nitric acid until a neutral $\mathrm{pH}$ was detected. The wet, oxidized nanotubes were placed into scintillation vials without caps to dry in the oven at $100^{\circ} \mathrm{C}$.

Approximately $\sim 0.01 \mathrm{~g}$ of the dry, oxidized MWCNTs were suspended in $10 \mathrm{~g}$ of a $50 / 50(\mathrm{v} / \mathrm{v} \%)$ solution of pure anhydrous IPA (Technical Grade, $>99.8 \%$, Techspray)/ultra-pure water. See Table SI 1 for specific concentrations. The mixture was suspended via tip sonication for five minutes at $50 \%$ power. The $0.1 \%$ (wt $\%$ ) suspensions were stored in capped scintillation vials, wrapped with Parafilm for later use. The suspensions were bath sonicated for one minute prior to use in the experiments described below. The presence of bound negative charge was confirmed by measuring the electrophoretic mobility with a Malvern Zetasizer Nano ZS. The electrophoretic mobility of MWCNTs was measured following sample dilution, $5 \mathrm{~min}$ of tip sonication, and then centrifugation to separate MWCNT bundles from dispersed tubes. Fig. SI - 1 shows the electrophoretic mobility of the MWCNTs. Measurements summarized in Fig. SI - 1 suggest that MWCNTs bear some negative charge prior to modification, which then increased following $>1 \mathrm{~h}$ of oxidative treatment. Note that oxidative treatment with nitric acid may shorten the MWCNTs and bundles. Tip sonication was conducted for identical time and power following oxidative treatment to smooth variations in length that may result from oxidative treatment. However, systematic changes in MWCNT length may contribute to the results presented herein that show effects of treatment duration.

\subsection{Surface pressure measurements}

A NIMA Technology Langmuir-Blodgett trough was used to measure the surface pressure of a MWCNT laden air/water interface. After thorough washing of the trough and barriers with ethanol and acetone, and several rinses with ultra-pure water $(>18.2 \mathrm{M} \Omega \mathrm{cm})$, the trough was filled with $70 \mathrm{~mL}$ of ultra-pure water, and the barriers were coupled to the belt and placed at the interface such that the area between them was $75 \mathrm{~cm}^{2}$. A microbalance coupled to a $0.5 \mathrm{~mm}$ radius platinum rod was utilized as the Wilhelmy sensor. The rod was lightly rinsed in ethanol, then oxidized with a Bunsen burner for $\sim 15-20$ s to enhance wetting of the rod. The clean rod was then hung from the microbalance. 

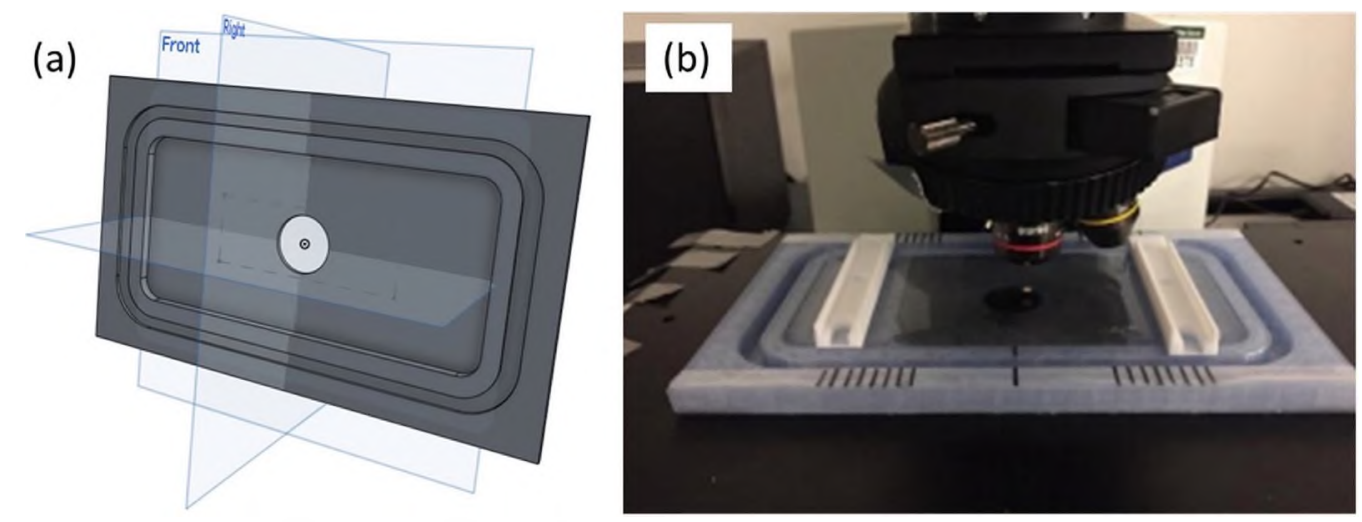

Fig. 1. (A) Computer-aided drawing and (B) utilization of custom 3D printed trough for optical microscopy measurements.

Surface pressure was zeroed prior to lowering the rod into the clean interface. The resulting pressure reading was checked against the surface tension of ultra-pure water to ensure the system's cleanliness. The cleaning procedure was repeated if the measured surface pressure was not within $+/-1 \mathrm{mN} / \mathrm{m}$ of the tabulated surface pressure for water at the specific temperature. Next, $300 \mu \mathrm{L}$ of MWCNT sample, suspended in IPA/Water and containing an initial MWCNT mass $\mathrm{m}_{0}$, was placed dropwise at random locations on the interface between the barriers. The barriers were compressed at a rate of $7 \mathrm{~cm}^{2} / \mathrm{min}$ to a minimum surface area of $25 \mathrm{~cm}^{2}$ and expanded back to the initial area of $75 \mathrm{~cm}^{2}$ for three cycles. A second dilatational measurement was conducted consisting of a step compression and long relaxation. The trough was set up in the same way as described above, but with the barriers compressed from $75 \mathrm{~cm}^{2}$ to $70 \mathrm{~cm}^{2}$ over a $30 \mathrm{~s}$ window. The surface pressure was measured during the compression and subsequent relaxation for one hour.

\subsection{Imaging of compressions and deposited MWCNTs}

A custom 3D printed trough with dimensions identical to the NIMA trough dimensions was designed to fit onto the microscope stage under the objectives to view the air/water interface laden with MWCNTs (see Fig. 1). Images were acquired using cellSens dimensions software in conjunction with an Olympus optical microscope and mounted Hamamatsu Orca R2 digital camera. The barriers from the NIMA trough were manually moved to marked increments from $75 \mathrm{~cm}^{2}$ to $25 \mathrm{~cm}^{2}$ to compress the interface. Individual MWCNTs were observed with a FEI/ Inspect F50 Field Emission Scanning Electron Microscope (SEM). SEM samples were prepared by removing a silicon substrate (University Wafer, Boston, MA) upward through the sub-phase at an angle of $90^{\circ}$ (perpendicular to the sub-phase). In an effort to obtain uniform deposition, the withdrawal rate of the substrate was coordinated with the compression rate of the barriers during deposition. A MWCNT film that uniformly covered the entire substrate was obtained when the relative barrier and substrate velocities were each $1 \mathrm{~cm} / \mathrm{min}$. Given that the trough width was $7 \mathrm{~cm}$, the corresponding area compression rate of the barriers was $7 \mathrm{~cm}^{2} / \mathrm{min}$. This compression rate, which was close to work on similar systems [26], was used for the surface pressure measurements.

\section{Results and discussion}

\subsection{Interfacial dispersion, film formation, and buckling}

MWCNTs were dispersed at an air/water interface. Initially, films dispersed well to fill the entire available interfacial area of $75 \mathrm{~cm}^{2}$. Barrier compression commenced immediately at a rate of $7 \mathrm{~cm}^{2} / \mathrm{min}$ to the final area of $25 \mathrm{~cm}^{2}$. Fig. 2 shows optical micrographs of the interface at maximum compression and surface coverage for untreated, $2 \mathrm{~h}$, and $6 \mathrm{~h}$ treated films. The interface laden with MWCNTs that did not undergo oxidative treatment buckled at maximum compression, while no buckling was observed from the interface laden with MWCNTs that had undergone $6 \mathrm{~h}$ of oxidative treatment. Buckling corresponded to a plateau of the surface pressure near its maximum value (presented later in article). Deflection or buckling of the interface is possible because surface tension approaches a minimum value at the plateau in surface pressure. MWCNT films showed a systematic decrease in the amount of buckling as the amount of oxidative treatment increased from none to $6 \mathrm{~h}$.

Densified MWCNT films were then deposited onto silicon chips for observation with SEM. MWCNTs were heterogeneous, with most particles grouped with others in bundles (see Fig. 3). In addition, individual MWCNTs were not observed to be significantly physically altered by the treatment process. Quantitative characterization of the MWCNT was not conducted. The industrial grade MWCNTs were highly polydisperse material, which was not amenable to typical characterization techniques to obtain a reasonable average length and diameter. Bundling in these samples likely originated from the strong van der Waals attraction in addition to capillary attraction during SEM sample preparation. Particles deposited on a substrate and dispersed in an evaporating fluid will experience capillary attraction when the fluid level reaches the particles. Small patches of alignment appear in the images, though there does not appear to be a correlation between the patchy alignment and trough area or modification time. Non-discrete layers of tubes appear in the microstructure, rather than a single monolayer, indicating that the interfacial layer of MWCNT has a percolated and multilayer structure following compression, with a significant number of particle/bundle contacts.

Following maximum compression, the barrier expanded at the rate of $7 \mathrm{~cm}^{2} / \mathrm{min}$, returning to the initial area of $75 \mathrm{~cm}^{2}$. The MWCNT film relaxed during the expansion until the critical area $A_{C}$, which was defined as the location of the initial plateau in surface pressure. This plateau in surface pressure corresponded to the area at which the MWCNT film detached from the barriers. The barriers continued to expand to the initial area, with a 'densified' film of area $A_{C}$ situated in the center of the trough. The densified film persisted for two additional compression and expansion cycles. The densified film remained intact, reattaching and detaching on each subsequent compression and expansion cycles, respectively. The critical area was recorded for each of the MWCNT samples on the first expansion cycle. The critical area was used to calculate the critical surface coverage $\Gamma_{C}=m_{0} / A_{G}$, defined as the value of the surface concentration at the critical surface area. The critical surface coverage ratio is then defined as the quotient of the critical and initial surface coverage $\Theta=\Gamma_{C} / \Gamma_{0}$. A MWCNT film that expands to fill the initial area following a compression will have a value of $\Theta=1$, while a MWCNT film densified beyond that of the initial film will have a value $\Theta>1$. Films that do not relax to $\Theta=1$ are those that retain particle/particle contacts that were induced during compression. Larger values of $\Theta$ indicate longer-lived or more frequent particle/ 

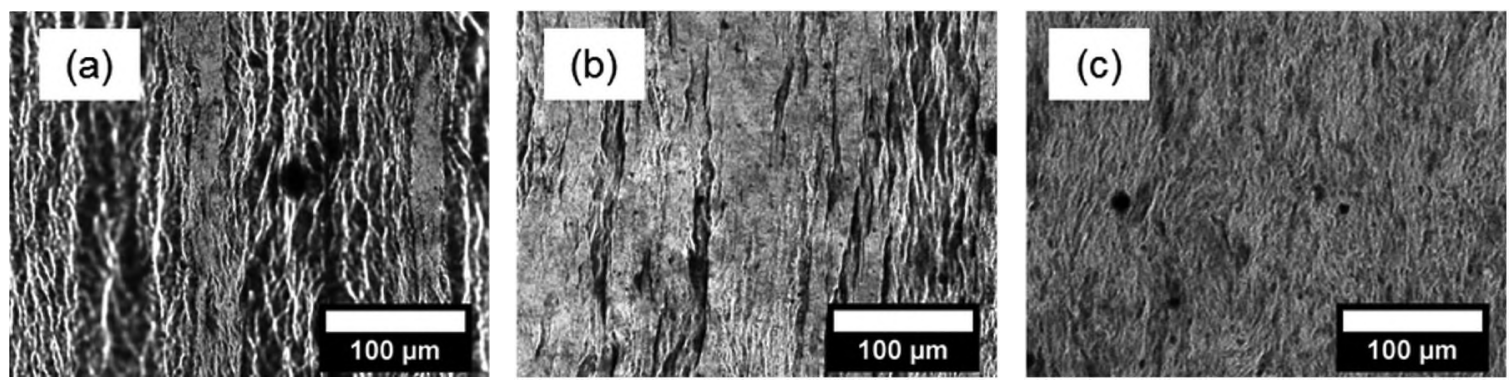

Fig. 2. Optical micrographs of an air/water interface laden with MWCNTs when compressed to $25 \mathrm{~cm}^{2}$. Significant buckling was observed for (a) non-treated MWCNTs, (b) some buckling for MWCNTs treated for $2 \mathrm{~h}$, and (c) no buckling for MWCNTs treated for $6 \mathrm{~h}$.

particle contacts in the film. The critical surface coverage ratio systematically increased with oxidative treatment in the presence of IPA (see Fig. 4).

\subsection{Mechanics of the MWCNT laden interface}

Three cycles of surface compression and expansions were conducted for MWCNT samples at room temperature and $25^{\circ} \mathrm{C}$. The surface pressure increased monotonically with a shallow slope on the initial compression, during which the film densified as described in the previous section. Fig. 5(a) shows that the initial shallow slope of surface pressure increased to the minimal area, corresponding to the maximum surface coverage. The shallow slope measured on the first compression was observed for all samples and was characteristic of the densification of the initially well dispersed MWCNT film. The expansion slope was far steeper. Surface pressure continued to decrease during the expansion part of the cycle, until the film detached from the barriers at the critical area. Surface pressure was flat as the barriers continued to expand beyond the critical area because the barriers no longer were deforming the film. Subsequent compression/expansion cycles (Fig. 5(b) and (c)) had behavior that was characteristic of the densified MWCNT film. Once the barriers were in contact with the film, the surface pressure increased (decreased) approximately linearly as the area decreased (increased).

Following densification, the surface pressure was an indirect measure of the stiffness of the solid-like MWCNT film. Gibbs elasticity was utilized to quantify the MWCNT film stiffness. Gibbs elasticity can be rewritten in terms of surface pressure by replacing the change in the surface tension with the negative change in the surface pressure and is approximated by $\kappa=-\frac{d \Pi}{d \ln (A)}$, where $\Pi$ is the surface pressure $[26,29,30]$. A stiffer film will have a higher value of $x$ and a correspondingly steeper slope of surface pressure vs. $\ln (\mathrm{A})$. Fig. 6 shows the Gibbs elasticity during expansion increased with increased oxidative treatment. Note, however, that Gibbs elasticity also increased with each successive compression cycle. The increase in film stiffness appears to be an indirect consequence of the oxidative treatment, with the
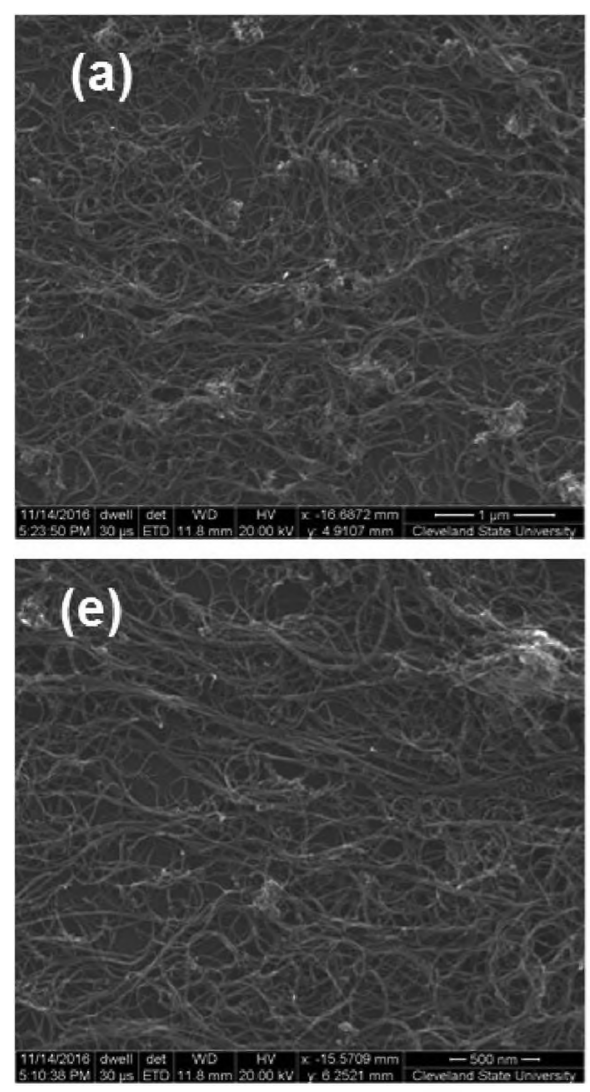
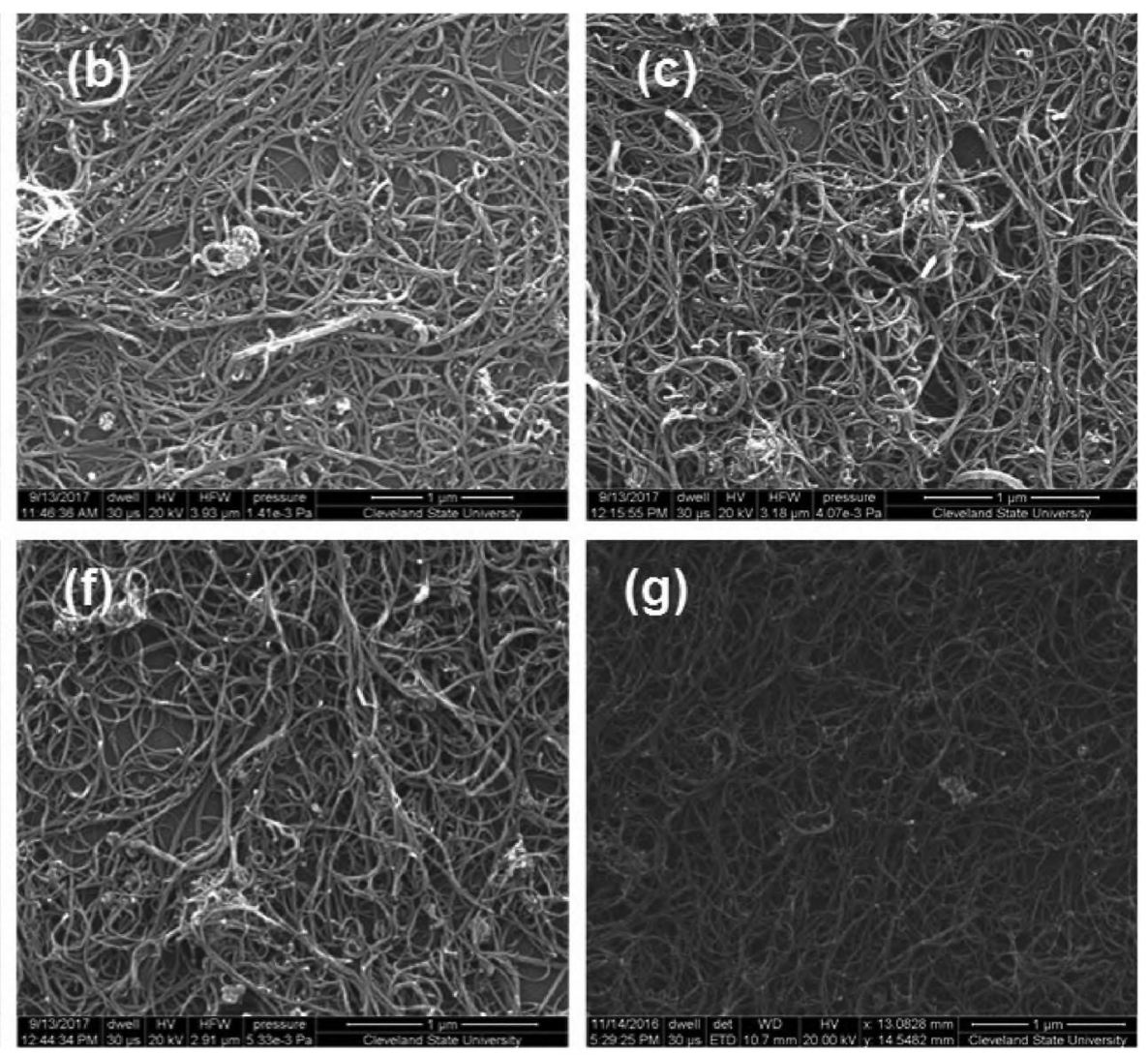

Fig. 3. Scanning electron microscope (SEM) images of industrial grade multiwalled carbon nanotubes (MWCNT) with (a) 0 min., (b) 40 min., (c) 1 hr., (d) 2 hrs., (e) 4 hrs., and (f) 6 hrs. treatment. Films were deposited when compressed to $25 \mathrm{~cm}^{2}$ from an air/water interface. The MWCNTs films were not comprised of organized tubes, but rather bundles with a high level of percolation and particle contacts. 


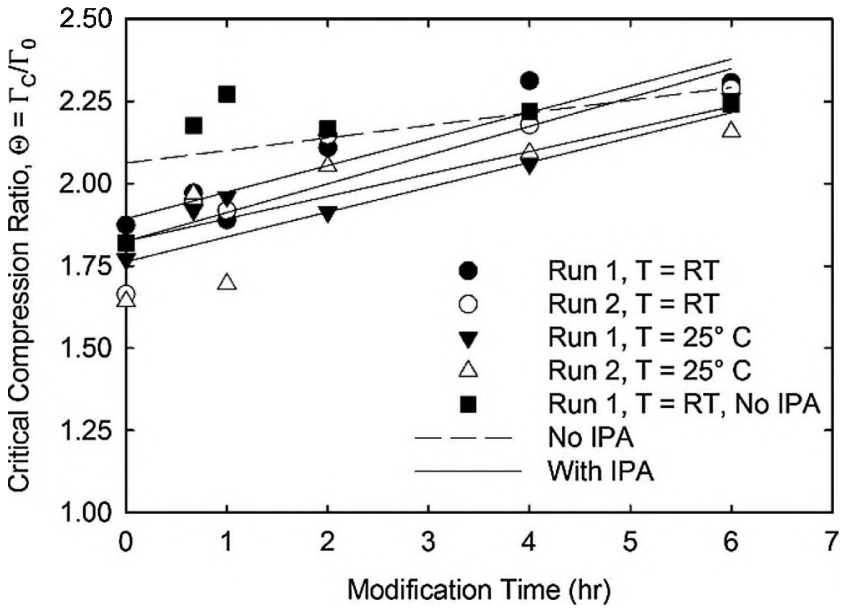

Fig. 4. Critical compression ratio for varying lengths of oxidative treatment and in the presence and absence of isopropyl alcohol (IPA). Critical compression ratio increased with increasing oxidative treatment in the presence of IPA, but was a weak function of oxidative treatment in the absence of IPA.

compressive densification history of the MWCNT film more directly impacting stiffness.

A direct comparison between film densification and stiffness was made by plotting $\kappa$ as a function of $\Theta$. This plot captured features of the compressive history. Fig. 7 shows the film stiffness increased with critical compression ratio when considering data from multiple oxidative treatments, compression cycles, with and without IPA. Recall that a large critical compression is indicative of a denser film. Thus, increasing stiffness corresponded to a film that had achieved a higher density of particle/particle interactions. These results suggest that MWCNTs exposed to longer oxidative treatments and in the presence of IPA were able to achieve a film with more and/or stronger particle/particle contacts exhibiting higher stiffness.

Results summarized above are those from experiments where compressions were immediately conducted prior to allowing the small volume $(\sim 150 \mu \mathrm{L})$ of isopropyl alcohol (IPA) from completely evaporating. A second set of experiments were conducted where IPA was permitted to evaporate for one hour prior to measurement to determine the impact of IPA on the densification process and the resulting stiffness of the film. The surface tension of water following the addition of $150 \mu \mathrm{L}$ of IPA in the absence of MWCNTs was measured to decay to $<1.6 \mathrm{mN} / \mathrm{m}$ after $40 \mathrm{~min}$. Based on these results, this volume of IPA was expected to evaporate after $60 \mathrm{~min}$. Fig. 4 shows the critical compression ratio for these samples. There was an initial jump in the critical compression ratio for MWCNTs as a function of oxidative treatment ( $\sim 1 \mathrm{~h}$ treatment) in the absence of IPA and also densification on subsequent cycles. Further, as shown in Fig. 6 , there was a reduced effect of oxidative treatment on the stiffness of the MWCNT layer in the absence of IPA. The stiffness as a function of oxidative treatment remained flat or decreased for samples where IPA was permitted to evaporate. As noted, there was still densification on subsequent cycles in the no IPA case. Fig. 7 shows $\kappa$ vs. $\Theta$ obtained in the absence of IPA. These data show the same trend as in the cases with IPA. The MWCNT film was able to achieve a densified state, which had a stiffness that increased with increasing film density. Thus, these data suggest that film densification was the primary contributing factor for generating stiffness.

\subsection{Microstructural relaxation and physical picture}

The measured surface pressure is related to the surface stress experienced by the film [31]. The stress has two primary contributions: a thermodynamic surface tension and a microstructural surface stress. These contributions can be separated by conducting a variety of interfacial rheology measurements, including step compressions [32]. For a
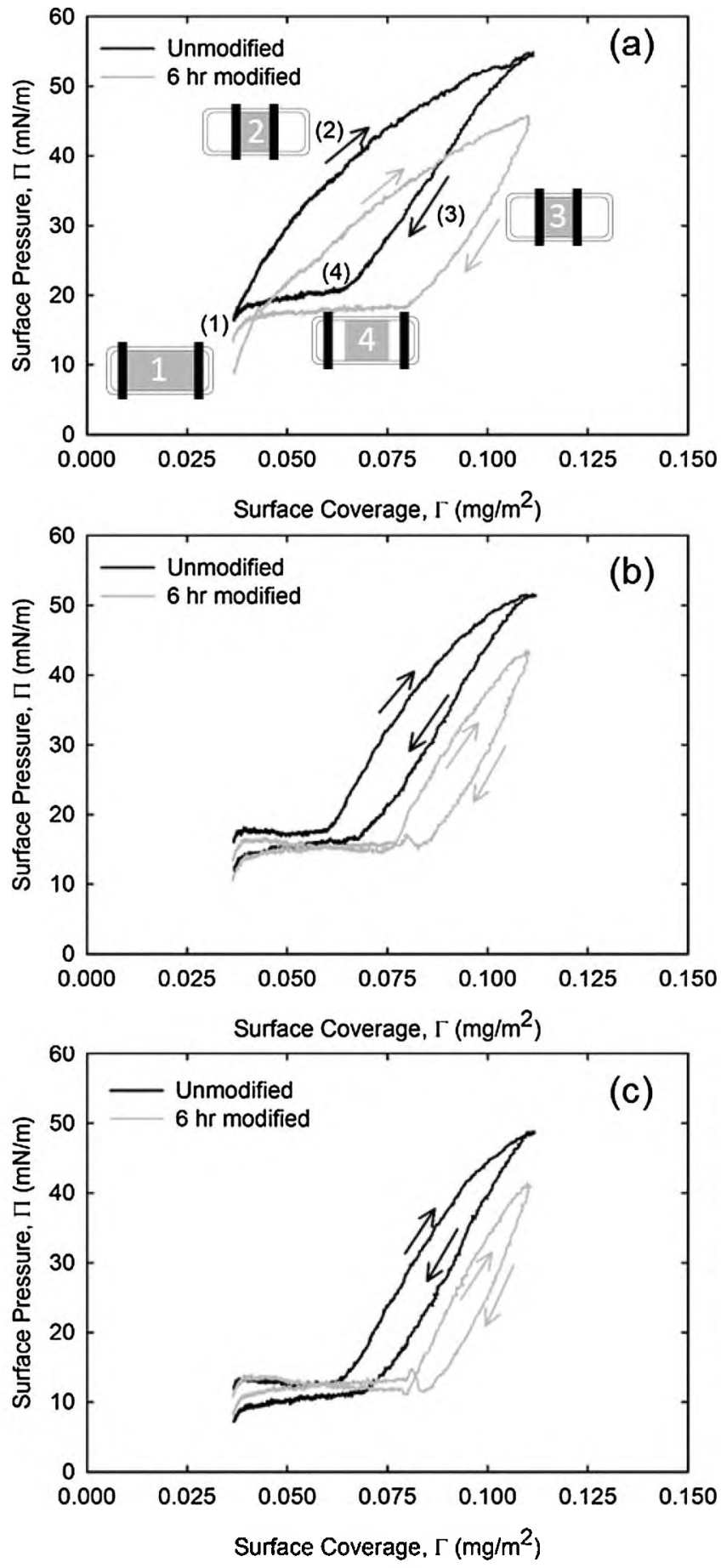

Fig. 5. Surface pressure compression and expansion cycles for untreated and treated MWCNTs for the (a) first, (b) second, and (c) third cycle. The surface pressure had an initial shallow slope, followed by a steep slope upon expansion. The shallow slope is characteristic of the initially well dispersed MWCNTs being densified into a film. Subsequent slopes for expansion and compression are steep with a flat portion. The film detaches from the barriers; when detached, the change in surface pressure with respect to the area is zero. The surface pressure measurements also show have a plateau for unmodified MWCNTs at maximum compression, which corresponds to the presence of buckling as documents in Fig. 2.

typical step compression measurement, the surface pressure is measured as the barriers are stepped to a new area. Following a spike in surface pressure resulting from the step change in area, the interface is allowed to relax. The steady-state surface pressure (as time $\rightarrow \infty$ ) is 

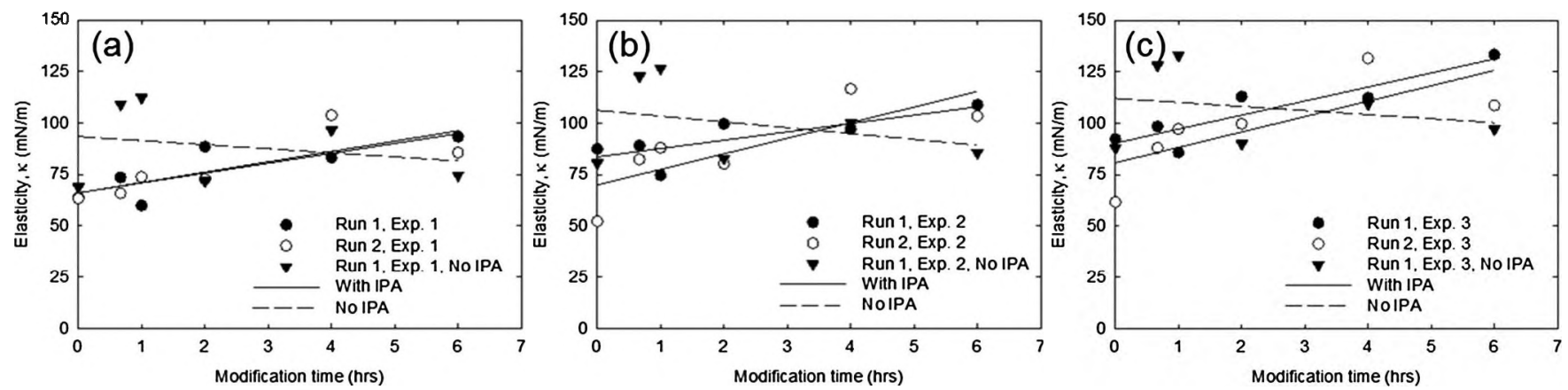

Fig. 6. Gibbs elasticity $\kappa$ for MWCNTs of varying durations of oxidative treatment and in the presence and absence of isopropyl alcohol (IPA) for (a) Expansion (Exp.) one, (b) Exp. two, and (c) Exp. three. Run 1 and Run 2 refer to repeats of the same experiment. A larger value of $\kappa$ indicates the film was stiffer. MWCNTs that had been treated longer in an oxidizing environment formed a stiffer film in the presence of IPA. However, the enhanced stiffness appears to depend more strongly on compression history than oxidative treatment or IPA.

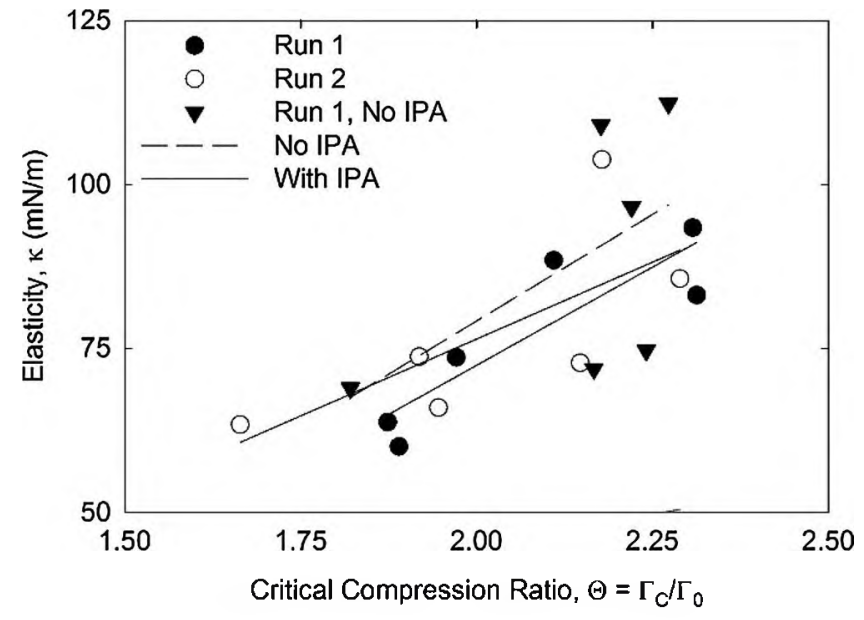

Fig. 7. Gibbs elasticity $\kappa$ as a function of critical compression ratio. Although the MWCNT samples displayed differences in stiffness depending on the presence of residual IPA, all samples behaved similarly when considering $\mathrm{k}$ as a function of critical compression ratio. The origin of differences in stiffness was in the variation in the level of interaction densification achieved by the MWCNT film.

equal to the thermodynamic surface tension, which depends only on surface coverage. The microstructural contribution to the surface pressure is the difference between the spike and the long time value.

Fig. 8 shows the long time relaxations for films of varying oxidative treatments in the presence of IPA. The data for each modification time can be more easily compared by dividing the surface pressure values of each run by their respective maximum surface pressures after compression such that all the relaxations start at a scaled surface pressure of 1. Note that data for a film of MWCNTs that had been modified for $1 \mathrm{~h}$ shows the fastest relaxation. However, as shown in the raw data (see Fig. SI 2), films comprised of MWCNTs with modification times of $4 \mathrm{~h}$ and $6 \mathrm{~h}$ had notably longer relaxation times, while the remaining four samples $(0 \mathrm{~h}, 40 \mathrm{~min}, 1 \mathrm{~h}$, and $2 \mathrm{~h}$ ) behaved similarly at low surface pressure. Scaling of these data amplified smaller differences in surface pressure, especially for the four modification times that all behaved similarly. A linear dependence between surface pressure and the square root of time indicates a diffusive microstructural relaxation (see Fig. 8) [32]. One of two effects can be inferred from the slopes in the graph. Either long relaxation times or larger equilibrium surface pressures may be indicated by the shallow slopes. Given that no plateau was observed in an experimentally accessible time (prior to evaporation effects), these results show MWCNT films have longer relaxation times for increasing treatment duration.

In the case of a step compression in the presence of IPA, MWCNT

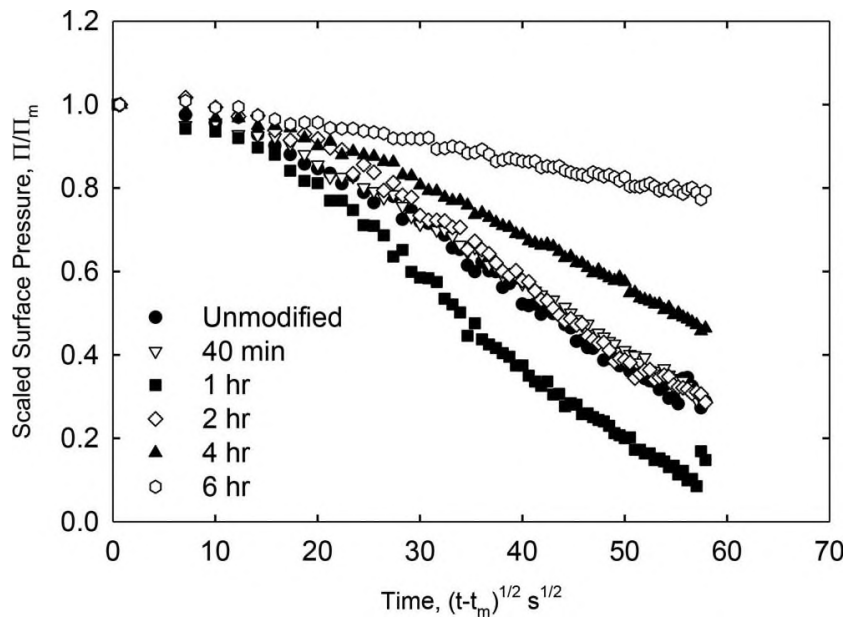

Fig. 8. Long time relaxations for industrial grade MWCNTs of varying extents of oxidative treatment in the presence of IPA. The MWCNT that had undergone the longest oxidative treatment had the slowest relaxation, again indicating that the densified film formed from this material had the strongest and/or most particle-cluster contacts. Recall that the MWCNT with the longest oxidative treatment was measured to be the stiffest film. Scaled data for a film of MWCNTs that had been modified for 1 h shows the fastest relaxation. Fig. SI 2 shows a grouping of behaviors, where films comprised of MWCNTs with modification times of $4 \mathrm{~h}$ and $6 \mathrm{~h}$ had long relaxation times, while the remaining four samples $(0 \mathrm{~h}, 40 \mathrm{~min}, 1 \mathrm{~h}$, and $2 \mathrm{~h}$ ) behaved similarly with faster relaxation. The maximum surface pressure used to scale each curve was $16.0 \mathrm{mN} / \mathrm{m}$ (unmodified), $19.6 \mathrm{mN} / \mathrm{m}$ (40 min.), $17.4 \mathrm{mN} / \mathrm{m}$ (1 h.), $18.2 \mathrm{mN} / \mathrm{m}$ ( 2 h.), $22.7 \mathrm{mN} / \mathrm{m}$ ( 4 h.), and $20.6 \mathrm{mN} / \mathrm{m}(6 \mathrm{~h}$.).

with longer oxidative treatment relaxed more slowly when compared to films with less treatment. The presence of IPA allowed MWCNTs with longer oxidative treatment to be wetted and subsequently form films with more and/or stronger particle-cluster contacts, which will take longer to relax. In the absence of IPA, the MWCNT of varying oxidative treatments behave roughly the same and any densification is simply a consequence of the transient microstructural development during IPA evaporation prior and subsequent compression.

These results show the primary factor for influencing film stiffness is compressive history, but there are more nuanced effects from particle wettability, residual alcohol, and aging. An IPA/water solution will lower the surface tension of water [33], thereby allowing for the liquid sub-phase to more easily wet the oxidized MWCNTs via interfacial deformation. Partially-wetted (relative to non-wetting) MWCNTs will adsorb more strongly to the interface, potentially inducing greater interfacial deformation and more significant capillary attraction between particles and bundles within the film. However, the transient wetting of a particle near an interface is known to be highly variable and 
potentially slow. Other work on model systems has shown long time aging of the contact line [34]. In the work described herein, the MWCNTs in the presence and absence of residual alcohol were added to the interface $\sim$ one min and one hour, respectively, prior to initiating a compression. Thus, MWCNT films in the absence of residual alcohol had relaxed for an hour prior to compression, which could facilitate wetting of particles regardless of oxidative treatment and may help to explain the weaker dependence of treatment time in the absence of residual alcohol.

Loss of MWCNT to the sub-phase may also contribute to changes in mechanics as a function of IPA and modification time. The presence of MWCNTs in the sub-phase was checked by removing $40 \mu \mathrm{L}$ aliquots following compressions and subsequently measuring the dried mass with a microbalance of sensitivity $\sim 0.1 \mu \mathrm{g}$. No dried mass was detected with the microbalance. Using the known volume and the balance sensitivity, it was determined that no more than $6 \%$ of MWCNT mass could have desorbed from the interface. Although this loss of MWCNTs may have contributed to the changes in mechanics, the typical change in critical coverage reported herein was $-30-40 \%$ as a function of modification time. Thus, changes solely in the surface coverage could not account for changes in the critical coverage in these instances. Additional dilatational measurements with systematically varied amounts of IPA need to be conducted to delineate these effects. These results and associated physical picture suggest that when formulating multiphase materials stabilized by MWCNTs, there is a significant and potential synergistic effect with the particles' surface chemistry, compressive history, and aging of the film.

\section{Conclusions}

The properties of an air/water interface laden with industrial grade MWCNTs were measured for varying duration of oxidative treatment and in the presence and absence of IPA. MWCNTs dispersed well at an air/water interface, with the initial compression forming a densified film that remained for the subsequent compression/expansion cycles. MWCNT films with longer oxidative treatments were measured to be stiffer than the same material with shorter oxidative treatment. The enhanced stiffness was manifested in the lack of buckling at the smallest area and correspondingly largest surface coverage. IPA appeared to enhance densification of particle/particle interactions in the film depending on the length of oxidative treatment of the MWCNT sample, whereas there was a weaker effect on the film mechanics in absence of IPA. Long time relaxations depending linearly on the square root of time supported the physical picture that MWCNTs with longer oxidative treatments in the presence of IPA formed a film with more and/or stronger particle-cluster contacts, although there was a confounding effect of aging that may play a role. These results suggest that not only does oxidative treatment and liquid composition play a role in MWCNT film mechanics, but also aging and compressive history.

\section{Author contributions}

The manuscript was written through contributions of all authors. All authors have given approval to the final version of the manuscript.

\section{Acknowledgements}

This work was supported by the Cleveland State University Office of Research Startup Fund and also the Undergraduate Summer Research Award Program. We acknowledge Professor Joseph R. Samaniuk (Department of Chemical and Biological Engineering, Colorado School of Mines) and Professor Yakov Lapitsky Department of Chemical Engineering, University of Toledo) for helpful discussions. We also acknowledge Professor Jessica Bickel (Department of Physics) and Aidin Rashidi (Department of Chemical and Biomedical Engineering) for assistance in SEM imaging. We also acknowledge Professor Jorge Gatica
(Department of Chemical and Biomedical Engineering) for assistance with the microbalance measurement.

\section{Appendix A. Supplementary data}

Supplementary material related to this article can be found, in the online version, at doi: https://doi.org/10.1016/j.colsurfa.2018.04.062.

\section{References}

[1] M.F.L. De Volder, S.H. Tawfick, R.H. Baughman, A.J. Hart, Carbon nanotubes: present and future commercial applications, Science (80-.) 339 (2013) 535-539, http://dx.doi.org/10.1126/science.1222453.

[2] M. Engel, J.P. Small, M. Steiner, M. Freitag, A.A. Green, M.C. Hersam, P. Avouris, Thin film nanotube transistors based on self-assembled, aligned, semiconducting carbon nanotube arrays, ACS Nano 2 (2008) 2445-2452, http://dx.doi.org/10. 1021/n11800708w.

[3] J.E. Trancik, S.C. Barton, J. Hone, Transparent and catalytic carbon nanotube films, Nano Lett. 8 (2008) 982-987, http://dx.doi.org/10.1021/n1071945i.

[4] C.J. Gannon, P. Cherukuri, B.I. Yakobson, L. Cognet, J.S. Kanzius, C. Kittrell, R.B. Weisman, M. Pasquali, H.K. Schmidt, R.E. Smalley, S.A. Curley, Carbon nanotube-enhanced thermal destruction of cancer cells in a noninvasive radiofrequency field, Cancer 110 (2007) 2654 2665, http://dx.doi.org/10.1002/cncr. 23155.

[5] M. Yu, Strength and breaking mechanism of multiwalled carbon nanotubes under tensile load, Science (80-.) 287 (2000) 637-640, http://dx.doi.org/10.1126/ science. 287.5453.637.

[6] T.W. Ebbesen, H.J. Lezec, H. Hiura, J.W. Bennett, H.F. Ghaemi, T. Thio, Electrical conductivity of individual carbon nanotubes, Nature 382 (1996) 54-56, http://dx. doi.org/10.1038/382054a0.

[7] T.K. Leeuw, R.M. Reith, R.A. Simonette, M.E. Harden, P. Cherukuri, D.A. Tsyboulski, K.M. Beckingham, R.B. Weisman, Single-walled carbon nanotubes in the intact organism: Near-IR imaging and biocompatibility studies in Drosophila, Nano Lett. 7 (2007) 2650-2654, http://dx.doi.org/10.1021/n10710452.

[8] P. Cherukuri, S.M. Bachilo, S.H. Litovsky, R.B. Weisman, Near-Infrared fluorescence microscopy of single-walled carbon nanotubes in phagocytic cells, J. Am. Chem. Soc. 126 (2004) 15638-15639, http://dx.doi.org/10.1021/ja0466311.

[9] X. Li, J.F. Gilchrist, Large-Area nanoparticle films by continuous automated Langmuir-Blodgett assembly and deposition, Langmuir 32 (2016) 1220-1226, http://dx.doi.org/10.1021/acs.langmuir.5b03760.

[10] M. Bardosova, M.E. Pemble, I.M. Povey, R.H. Tredgold, The langmuir-blodgett approach to making colloidal photonic crystals from silica spheres, Adv. Mater. 22 (2010) 3104-3124, http://dx.doi.org/10.1002/adma.200903708.

[11] K. Muramatsu, M. Takahashi, K. Tajima, K. Kobayashi, Two-Dimensional assemblies of colloidal $\mathrm{SiO} 2$ and $\mathrm{TiO} 2$ particles prepared by the Langmuir-Blodgett technique J. Colloid Interface Sci. 242 (2001) 127-132, http://dx. doi.org/10.1006/jcis.2001. 7784.

[12] X. Li, L. Zhang, X. Wang, I. Shimoyama, X. Sun, W.K. Seo, H. Dai, LangmuirBlodgett assembly of densely aligned single-walled carbon nanotubes from bulk materials, J. Am. Chem. Soc. 129 (2007) 4890-4891, http://dx.doi.org/10.1021/ ja071114e.

[13] C.L. Wirth, M. De Volder, J. Vermant, Fabrication of planar colloidal clusters with template-assisted interfacial assembly, Langmuir 31 (2015), http://dx.doi.org/10. $1021 / 1 \mathrm{a} 504383 \mathrm{~m}$

[14] B.P. Binks, T.S. Horozov (Eds.), Colloidal Particles at Liquid Interfaces, 1st ed., Cambridge University Press, Cambridge, 2006.

[15] B.P. Binks, S.O. Lumsdon, Pickering emulsions stabilized by monodisperse latex particles: effects of particle size, Langmuir 17 (2001) 4540-4547, http://dx.doi org/10.1021/1a0103822.

[16] A. Stocco, E. Rio, B.P. Binks, D. Langevin, Aqueous foams stabilized solely by particles, Soft Matter 7 (2011) 1260, http://dx.doi.org/10.1039/c0sm01290d.

[17] L. Botto, E.P. Lewandowski, M. Cavallaro, K.J. Stebe, Capillary interactions between anisotropic particles, Soft Matter 8 (2012) 9957, http://dx.doi.org/10.1039/ c2sm25929j.

[18] B. Madivala, S. Vandebril, J. Fransaer, J. Vermant, Exploiting particle shape in solid stabilized emulsions, Soft Matter 5 (2009) 1717, http://dx.doi.org/10.1039/ b816680c.

[19] G. Kaptay, N. Babcsán, Particle stabilized foams, Foam Eng. Fundam. Appl. 2000 (2012) 121-143, http://dx.doi.org/10.1002/9781119954620.ch7.

[20] E. Rio, W. Drenckhan, A. Salonen, D. Langevin, Unusually stable liquid foams, Adv. Colloid Interface Sci. 205 (2014) 74-86, http://dx.doi.org/10.1016/j.cis.2013.10. 023.

[21] B.P. Binks, T.S. Horozov, Aqueous foams stabilized solely by silica nanoparticles, Angew. Chem. 117 (2005) 3788-3791, http://dx.doi.org/10.1002/ange. 200462470.

[22] D. Pradilla, S. Simon, J. Sjoblom, J. Samaniuk, M. Skrzypiec, J. Vermant, Sorption and interfacial rheology study of model asphaltene compounds, Langmuir 32 (2016) 2900-2911, http://dx.doi.org/10.1021/acs.langmuir.6b00195.

[23] J.R. Samaniuk, E. Hermans, T. Verwijlen, V. Pauchard, J. Vermant, Soft-Glassy rheology of asphaltenes at liquid interfaces, J. Dispers. Sci. Technol. 36 (2015) 1444-1451, http://dx. doi.org/10.1080/01932691.2015.1022654.

[24] D.M. Sztukowski, H.W. Yarranton, Rheology of Asphaltene-Toluene/Water interfaces, Langmuir 21 (2005) 11651-11658, http://dx.doi.org/10.1021/la051921w. 
[25] S.R. Vora, B. Bognet, H.S. Patanwala, F. Chinesta, A.W.K. Ma, Surface pressure and microstructure of carbon nanotubes at an air-water interface, Langmuir 31 (2015) 4663-4672, http://dx.doi.org/10.1021/la504934x.

[26] L. Imperiali, K.H. Liao, C. Clasen, J. Fransaer, C.W. MacOsko, J. Vermant, Interfacial rheology and structure of tiled graphene oxide sheets, Langmuir 28 (2012) 7990-8000, http://dx.doi.org/10.1021/la300597n.

[27] D.M. Goggin, J.R. Samaniuk, Dynamics of pristine graphite and graphene at an airwater interface, AIChE J. (2018), http://dx.doi.org/10.1002/aic.16112.

[28] V. Datsyuk, M. Kalyva, K. Papagelis, J. Parthenios, D. Tasis, A. Siokou, I. Kallitsis, C. Galiotis, Chemical oxidation of multiwalled carbon nanotubes, Carbon N. Y. 46 (2008) 833-840, http://dx.doi.org/10.1016/j.carbon.2008.02.012.

[29] J. Berg, An Introduction to Interfaces and Colloids, 1st ed., World Scientific, 2010.

[30] G.G. Fuller, J. Vermant, Complex fluid-fluid interfaces: rheology and structure, Annu. Rev. Chem. Biomol. Eng. 3 (2012) 519-543, http://dx.doi.org/10.1146/ annurev-chembioeng-061010-114202.

[31] T. Verwijlen, L. Imperiali, J. Vermant, Separating viscoelastic and compressibility contributions in pressure-area isotherm measurements, Adv. Colloid Interface Sci. 206 (2014) 428-436, http://dx.doi.org/10.1016/j.cis.2013.09.005.

[32] A.J. Mendoza, E. Guzmản, F. Martínez-Pedrero, H. Ritacco, R.G. Rubio, F. Ortega, V.M. Starov, R. Miller, Particle laden fluid interfaces: dynamics and interfacial rheology, Adv. Colloid Interface Sci. 206 (2014) 303-319, http://dx.doi.org/10. $1016 /$ j.cis.2013.10.010.

[33] G. Vazquez, E. Alvarez, J.M. Navaza, Surface tension of alcohol Water + Water from 20 to 50. degree. C, J. Chem. Eng. Data. 40 (1995) 611-614, http://dx.doi org/10.1021/je00019a016.

[34] D.M. Kaz, R. McGorty, M. Mani, M.P. Brenner, V.N. Manoharan, Physical ageing of the contact line on colloidal particles at liquid interfaces, Nat. Mater. 11 (2011) 138-142, http://dx. doi.org/10.1038/nmat3190.

Post-print standardized by MSL Academic Endeavors, the imprint of the Michael Schwartz Library at Cleveland State University, 2019 